\title{
Some Characterizations of the Cobb-Douglas and CES Production Functions in Microeconomics
}

\author{
Xiaoshu Wang ${ }^{1}$ and $Y \mathbf{u ~ F u}^{2}$ \\ ${ }^{1}$ School of Investment and Construction Management, Dongbei University of Finance and Economics, Dalian 116025, China \\ ${ }^{2}$ School of Mathematics and Quantitative Economics, Dongbei University of Finance and Economics, Dalian 116025, China
}

Correspondence should be addressed to Yu Fu; yufudufe@gmail.com

Received 5 November 2013; Accepted 10 December 2013

Academic Editor: Grzegorz Lukaszewicz

Copyright (C) 2013 X. Wang and Y. Fu. This is an open access article distributed under the Creative Commons Attribution License, which permits unrestricted use, distribution, and reproduction in any medium, provided the original work is properly cited.

It is well known that the study of the shape and the properties of the production possibility frontier is a subject of great interest in economic analysis. Vîlcu (Vîlcu, 2011) proved that the generalized Cobb-Douglas production function has constant return to scale if and only if the corresponding hypersurface is developable. Later on, the authors A. D. Vîlcu and G. E. Vîlcu, 2011 extended this result to the case of CES production function. Both results establish an interesting link between some fundamental notions in the theory of production functions and the differential geometry of hypersurfaces in Euclidean spaces. In this paper, we give some characterizations of minimal generalized Cobb-Douglas and CES production hypersurfaces in Euclidean spaces.

\section{Introduction}

In microeconomics and macroeconomics, the production functions are positive nonconstant functions that specify the output of a firm, an industry, or an entire economy for all combinations of inputs. Almost all economic theories presuppose a production function, either on the firm level or the aggregate level. Hence, the production function is one of the most key concepts of mainstream neoclassical theories. By assuming that the maximum output technologically possible from a given set of inputs is achieved, economists always using a production function in analysis are abstracting from the engineering and managerial problems inherently associated with a particular production process; see [1].

In 1928, Cobb and Douglas [1] introduced a famous twofactor production function, nowadays called Cobb-Douglas production function, in order to describe the distribution of the national income by help of production functions. Twofactor Cobb-Douglas production function is given by

$$
Y=b L^{k} C^{1-k} \text {, }
$$

where $L$ denotes the labor input, $C$ is the capital input, $b$ is the total factor productivity, and $Y$ is the total production.

The Cobb-Douglas (CD) production function is especially notable for being the first time an aggregate or economy-wide production function was developed, estimated, and then presented to the profession for analysis. It gave a landmark change in how economists approached macroeconomics. The Cobb-Douglas function has also been applied to many other issues besides production.

The generalized form of the Cobb-Douglas production function is written as

$$
F\left(x_{1}, \ldots, x_{n}\right)=A x_{1}^{\alpha_{1}} \cdots x_{n}^{\alpha_{n}}
$$

where $x_{i}>0(i=1, \ldots, n), A$ is a positive constant, and $\alpha_{1}, \ldots, \alpha_{n}$ are nonzero constants.

In 1961, Arrow et al. [2] introduced another two-input production function given by

$$
Q=b\left(a K^{r}+(1-a) L^{r}\right)^{1 / r},
$$

where $Q$ is the output, $b$ the factor productivity, $a$ the share parameter, $K$ and $L$ the primary production factors, $r=(s-$ $1) / s$, and $s=1 /(1-r)$ the elasticity of substitution. Hence this is called constant elasticity of substitution (CES) production function $[3,4]$. 

by

The generalized form of CES production function is given

$$
F\left(x_{1}, \ldots, x_{n}\right)=A\left(\sum_{i=1}^{n} a_{i}^{\rho} x_{i}^{\rho}\right)^{\gamma / \rho},
$$

where $a_{i}, \gamma, A$, and $\rho$ are nonzero constants and $A, a_{i}>$ 0 . The CES production functions are of great interest in economy because of their invariant characteristic, namely, that the elasticity of substitution between the parameters is constant on their domains.

It is easy to see that the CES production function is a generalization of Cobb-Douglas production function, and both of them are homogeneous production functions.

Note that the production function $F$ can be identified with a production hypersurface in the $(n+1)$-dimensional Euclidean space $\mathbb{E}^{n+1}$ through the map $f: \mathbb{R}_{+}^{n} \rightarrow \mathbb{R}_{+}^{n+1}$,

$$
f\left(x_{1}, \ldots, x_{n}\right)=\left(x_{1}, \ldots, x_{n}, F\left(x_{1}, \ldots, x_{n}\right)\right) .
$$

In the case of $n$ inputs, we have a hypersurface and an analysis of the generalized CD and CES production functions from the point of view of differential geometry.

In [5], Vîlcu established an interesting link between some fundamental notions in the theory of production functions and the differential geometry of hypersurfaces. The author proved that the generalized Cobb-Douglas production function has constant return to scale if and only if the corresponding hypersurface is developable. The author jointly with A. D. Vîlcu further [6] generalized this result to the case of the generalized CES production function with $n$-inputs. For further study of production hypersurfaces, we refer the reader to Chen's series of interesting papers on homogeneous production functions, quasi-sum production models, and homothetic production functions [7-13] and G. E. Vîlcu and A. D. Vîlcu's paper [14].

The theory of minimal surfaces (hypersurfaces) is very important in the field of differential geometry. In this paper, we give further study of the generalized Cobb-Douglas and CES production functions as hypersurfaces in Euclidean space $\mathbb{E}^{n+1}$. In particular, we give some characterizations of the generalized Cobb-Douglas and CES production hypersurfaces under the minimality condition in Euclidean spaces.

\section{Some Basic Concepts in Theory of Hypersurfaces}

Each production function $F\left(x_{1}, \ldots, x_{n}\right)$ can be identified with a graph of a nonparametric hypersurface of an Euclidean $(n+$ 1)-space $\mathbb{E}^{n+1}$ given by

$$
f\left(x_{1}, \ldots, x_{n}\right)=\left(x_{1}, \ldots, x_{n}, F\left(x_{1}, \ldots, x_{n}\right)\right) .
$$

We recall some basic concepts in the theory of hypersurfaces in Euclidean spaces.

Let $M^{n}$ be a hypersurface in an $n+1$-dimension Euclidean space. The Gauss map $v$ is defined by

$$
v: M^{n} \longrightarrow S^{n} \subset \mathbb{E}^{n+1},
$$

which maps $M^{n}$ to the unit hypersphere $S^{n}$ of $\mathbb{E}^{n+1}$. The Gauss map is always defined locally, that is, on a small piece of the hypersurface. It can be defined globally if the hypersurface is orientable. The Gauss map is a continuous map such that $v(p)$ is a unit normal vector $\xi(p)$ of $M^{n}$ at point $p$.

The differential $d v$ of the Gauss map $v$ can be used to define an extrinsic curvature, known as the shape operator or Weingarten map. Since, at each point $p \in M^{n}$, the tangent space $T_{p} M$ is an inner product space, the shape operator $S_{p}$ can be defined as a linear operator on this space by the relation

$$
g\left(S_{p} u, w\right)=g(d v(u), w),
$$

where $u, w \in T_{p} M$ and $g$ is the metric tensor on $M^{n}$.

Moreover, the second fundamental form $h$ is related to the shape operator $S_{p}$ by

$$
g\left(S_{p} u, w\right)=g(h(u, w), \xi(p))
$$

for $u, w \in T_{p} M$.

It is well known that the determinant of the shape operator $S_{p}$ is called the Gauss-Kronecker curvature. Denote it by $G(p)$. When $n=2$, the Gauss-Kronecker curvature is simply called the Gauss curvature, which is intrinsic due to famous Gauss's Theorema Egregium. The trace of the shape operator $S_{p}$ is called the mean curvature of the hypersurfaces. In contrast to the Gauss curvature, the mean curvature is extrinsic, which depends on the immersion of the hypersurface. A hypersurface is called minimal if its mean curvature vanishes identically.

Denote the partial derivatives $\partial F / \partial x_{i}, \partial^{2} F / \partial x_{i} x_{j}, \ldots$, and so forth by $F_{i}, F_{i j}, \ldots$, and so forth. Put

$$
W=\sqrt{1+\sum_{i=1}^{n} F_{i}^{2}}
$$

Recall some well-known results for a graph of hypersurface (6) in $\mathbb{E}^{n+1}$ from $[7,15,16]$.

Proposition 1. For a production hypersurface of $\mathbb{E}^{n+1}$ defined by

$$
f\left(x_{1}, \ldots, x_{n}\right)=\left(x_{1}, \ldots, x_{n}, F\left(x_{1}, x_{n}\right)\right)
$$

One has the following.

(1) The unit normal $\xi$ is given by

$$
\xi=\frac{1}{W}\left(-F_{1}, \ldots,-F_{n}, 1\right) .
$$

(2) The coefficient $g_{i j}=g\left(\partial / \partial x_{i}, \partial / \partial x_{j}\right)$ of the metric tensor is given by

$$
g_{i j}=\delta_{i j}+F_{i} F_{j}
$$

where $\delta_{i j}=1$ if $i=j$, otherwise 0.

(3) The volume element is

$$
d V=\sqrt{g_{i j}} d x_{1} \wedge \cdots \wedge d x_{n} .
$$


(4) The inverse matrix $\left(g^{i j}\right)$ of $\left(g_{i j}\right)$ is

$$
g^{i j}=\delta_{i j}-\frac{F_{i} F_{j}}{W^{2}} \text {. }
$$

(5) The matrix of the second fundamental form $h$ is

$$
h_{i j}=\frac{F_{i j}}{W} .
$$

(6) The matrix of the shape operator $S_{p}$ is

$$
a_{i}^{j}=\sum_{k} h_{i k} g^{k j}=\frac{1}{W}\left(F_{i j}-\sum_{k} \frac{F_{i k} F_{k} F_{j}}{W^{2}}\right) .
$$

(7) The mean curvature $H$ is given by

$$
H=\frac{1}{n W}\left(\sum_{i} F_{i i}-\frac{1}{W^{2}} \sum_{i, j} F_{i} F_{j} F_{i j}\right) .
$$

(8) The Gauss-Kronecker curvature $G$ is

$$
G=\frac{\operatorname{det} h_{i j}}{\operatorname{det} g_{i j}}=\frac{\operatorname{det} F_{i j}}{W^{n+2}} .
$$

(9) The sectional curvature $K_{i j}$ of the plane section spanned by $\partial / \partial x_{i}$ and $\partial / \partial x_{j}$ is

$$
K_{i j}=\frac{F_{i i} F_{j j}-F_{i j}^{2}}{W^{2}\left(1+F_{i}^{2}+F_{j}^{2}\right)} .
$$

(10) The Riemann curvature tensor R satisfies

$$
g\left(R\left(\frac{\partial}{\partial x_{i}}, \frac{\partial}{\partial x_{j}}\right) \frac{\partial}{\partial x_{k}}, \frac{\partial}{\partial x_{l}}\right)=\frac{F_{i l} F_{j k}-F_{i k} F_{j l}}{W^{4}} .
$$

\section{Generalized Cobb-Douglas Production Hypersurfaces}

In this section, we give a nonexistence result of minimal generalized Cobb-Douglas production hypersurfaces in $\mathbb{E}^{n+1}$.

Theorem 2. There does not exist a minimal generalized CobbDouglas production hypersurface in $\mathbb{E}^{n+1}$.

Proof. Let $M$ be a generalized Cobb-Douglas production hypersurface in $\mathbb{E}^{n+1}$ given by a graph

$$
f\left(x_{1}, \ldots, x_{n}\right)=\left(x_{1}, \ldots, x_{n}, F\left(x_{1}, \ldots, x_{n}\right)\right) \text {, }
$$

where $F$ is the generalized Cobb-Douglas production function

$$
F\left(x_{1}, \ldots, x_{n}\right)=A x_{1}^{\alpha_{1}} \cdots x_{n}^{\alpha_{n}}
$$

Note that the generalized Cobb-Douglas production function (23) is homogeneous of degree $h=\sum_{i=1}^{n} \alpha_{i}$. It follows from (23) that

$$
F_{i}=\frac{\alpha_{i}}{x_{i}} F, \quad i=1, \ldots, n,
$$

$$
\begin{gathered}
F_{i i}=\frac{\alpha_{i}\left(\alpha_{i}-1\right)}{x_{i}^{2}} F, \quad i=1, \ldots, n, \\
F_{i j}=\frac{\alpha_{i} \alpha_{j}}{x_{i} x_{j}} F, \quad i \neq j, i, j=1, \ldots, n .
\end{gathered}
$$

By assumption, the production hypersurface is minimal; that is, $H=0$. Thus, by (18) and (10) we have

$$
\left(\sum_{i=1}^{n} F_{i i}\right)\left(1+\sum_{i=1}^{n} F_{i}^{2}\right)=\sum_{i, j} F_{i} F_{j} F_{i j}
$$

which reduces to

$$
\sum_{i=1}^{n} F_{i i}+\sum_{i, j}\left(F_{i}^{2} F_{j j}-F_{i} F_{j} F_{i j}\right)=0 .
$$

Note that $\sum_{i, j}\left(F_{i}^{2} F_{j j}-F_{i} F_{j} F_{i j}\right)=0$ for $i=j$. Hence (26) becomes

$$
\sum_{i=1}^{n} F_{i i}+\sum_{i \neq j}\left(F_{i}^{2} F_{j j}-F_{i} F_{j} F_{i j}\right)=0
$$

Substituting (24) into (27), we obtain

$$
\sum_{i=1}^{n} \frac{\alpha_{i}\left(\alpha_{i}-1\right)}{x_{i}^{2}}-F^{2} \sum_{i \neq j} \frac{\alpha_{i}^{2} \alpha_{j}}{x_{i}^{2} x_{j}^{2}}=0
$$

Differentiating (28) with respect to $x_{k}$, one has

$$
-\frac{2 \alpha_{k}\left(\alpha_{k}-1\right)}{x_{k}^{3}}-\frac{2 \alpha_{k}}{x_{k}} F^{2} \sum_{i \neq j} \frac{\alpha_{i}^{2} \alpha_{j}}{x_{i}^{2} x_{j}^{2}}+\frac{2 \alpha_{k}}{x_{k}} F^{2} \sum_{i=1}^{n} \frac{\alpha_{i} \alpha_{k}+\alpha_{i}^{2}}{x_{i}^{2} x_{k}^{2}}=0,
$$

which reduces to

$$
\frac{\alpha_{k}-1}{x_{k}^{2}}+F^{2} \sum_{i \neq j} \frac{\alpha_{i}^{2} \alpha_{j}}{x_{i}^{2} x_{j}^{2}}-F^{2} \sum_{i=1}^{n} \frac{\alpha_{i} \alpha_{k}+\alpha_{i}^{2}}{x_{i}^{2} x_{k}^{2}}=0 .
$$

Substituting (28) into (30), we have

$$
\frac{\alpha_{k}-1}{x_{k}^{2}}+\sum_{i=1}^{n} \frac{\alpha_{i}\left(\alpha_{i}-1\right)}{x_{i}^{2}}-F^{2} \sum_{i=1}^{n} \frac{\alpha_{i} \alpha_{k}+\alpha_{i}^{2}}{x_{i}^{2} x_{k}^{2}}=0 .
$$

Moreover, differentiating (31) with respect to $x_{l}(l \neq k)$, we get

$$
-\frac{2 \alpha_{l}\left(\alpha_{l}-1\right)}{x_{l}^{3}}+\frac{2 \alpha_{l} \alpha_{k}+2 \alpha_{l}^{2}}{x_{l}^{3} x_{k}^{2}} F^{2}-\frac{2 \alpha_{l}}{x_{l}} F^{2} \sum_{i=1}^{n} \frac{\alpha_{i} \alpha_{k}+\alpha_{i}^{2}}{x_{i}^{2} x_{k}^{2}}=0 .
$$


Combining (32) with (31) gives

$$
\frac{\alpha_{l}-1}{x_{l}^{2}}-\frac{\alpha_{k}+\alpha_{l}}{x_{l}^{2} x_{k}^{2}} F^{2}+\frac{\alpha_{k}-1}{x_{k}^{2}}+\sum_{i=1}^{n} \frac{\alpha_{i}\left(\alpha_{i}-1\right)}{x_{i}^{2}}=0 .
$$

Differentiating (33) with respect to $x_{l}$ again, one has

$$
\frac{\left(\alpha_{k}+\alpha_{l}\right)\left(\alpha_{l}-1\right)}{x_{l}^{3} x_{k}^{2}} F^{2}+\frac{\left(\alpha_{l}-1\right)\left(\alpha_{1}+1\right)}{x_{l}^{3}}=0,
$$

which yields either $\alpha_{l}=1(l \neq k)$ or

$$
\left(\alpha_{k}+\alpha_{l}\right) F^{2}=\left(\alpha_{l}+1\right) x_{k}^{2}
$$

Since $F$ is defined by (23) with nonzero constants $\alpha_{i}$ for $i=$ $1, \ldots, n$, it follows that (35) is impossible. Hence we have $\alpha_{l}=$ 1 .

Without loss of generality, we may choose $k=1$. In this case, $\alpha_{l}=1$ for $l \neq 1$ reduces to

$$
\alpha_{2}=\cdots=\alpha_{n}=1
$$

Therefore, (33) becomes

$$
-\frac{\alpha_{1}+1}{x_{1}^{2} x_{l}^{2}} F^{2}+\frac{\alpha_{1}^{2}-1}{x_{1}^{2}}=0
$$

The above equation implies that either $\alpha_{1}=-1$ or

$$
F^{2}=\left(\alpha_{1}-1\right) x_{l}^{2}
$$

It is easy to see that the latter case is impossible. At this moment, we obtain the function $F$ as follows:

$$
F\left(x_{1}, \ldots, x_{n}\right)=A x_{1}^{-1} x_{2} \cdots x_{n} .
$$

Consider (31) for $k \neq 1$, which turns into

$$
\frac{1}{x_{1}^{2}}-\frac{F^{2}}{x_{k}^{2}}\left(\frac{1}{x_{2}^{2}}+\cdots+\frac{1}{x_{n}^{2}}\right)=0
$$

Substituting (39) into (40), we have

$$
A^{2} x_{2}^{2} \cdots x_{n}^{2}\left(\frac{1}{x_{2}^{2}}+\cdots+\frac{1}{x_{n}^{2}}\right)=x_{k}^{2}
$$

In view of (41), by comparing the degrees of the left and the right of the equality we have $n=3$. Hence (41) becomes

$$
A^{2}\left(x_{2}^{2}+x_{3}^{2}\right)=x_{k}^{2}, \quad k=2,3,
$$

which is impossible since $A \neq 0$.

Therefore, there is not a minimal generalized CobbDouglas production hypersurface in $\mathbb{E}^{n+1}$. This completes the proof of Theorem 2 .

\section{Generalized CES Production Hypersurfaces}

In economics, goods that are completely substitutable with each other are called perfect substitutes. They may be characterized as goods having a constant marginal rate of substitution. Mathematically, a production function is called a perfect substitute if it is of the form

$$
F\left(x_{1}, \ldots, x_{n}\right)=\sum_{i=1}^{n} a_{i} x_{i}
$$

for some nonzero constants $a_{1}, \ldots, a_{n}$.

In the following, we deal with the case of minimal generalized CES production hypersurfaces in $\mathbb{E}^{n+1}$.

Theorem 3. An n-factor generalized CES production hypersurface in $\mathbb{E}^{n+1}$ is minimal if and only if the generalized CES production function is a perfect substitute.

Proof. Assume that $M$ is a generalized CES production hypersurface in $\mathbb{E}^{n+1}$ given by a graph

$$
f\left(x_{1}, \ldots, x_{n}\right)=\left(x_{1}, \ldots, x_{n}, F\left(x_{1}, \ldots, x_{n}\right)\right),
$$

where $F$ is the generalized CES production function

$$
F\left(x_{1}, \ldots, x_{n}\right)=A\left(\sum_{i=1}^{n} a_{i}^{\rho} x_{i}^{\rho}\right)^{\gamma / \rho},
$$

with $A, a_{i}>0$ and $x_{i}>0$. The generalized CES production function (45) is homogeneous of degree $\gamma$. By assumption that the production hypersurface is minimal, from (18) the minimality condition is equivalent to

$$
\left(\sum_{i=1}^{n} F_{i i}\right)\left(1+\sum_{i=1}^{n} F_{i}^{2}\right)=\sum_{i, j} F_{i} F_{j} F_{i j} .
$$

We define another minimal production hypersurface as

$$
\begin{gathered}
\tilde{f}\left(\lambda x_{1}, \ldots, \lambda x_{n}\right)=\left(\lambda x_{1}, \ldots, \lambda x_{n}, F\left(\lambda x_{1}, \ldots, \lambda x_{n}\right)\right), \\
\lambda \in \mathbb{R}^{+} .
\end{gathered}
$$

Since the CES production function is homogeneous of degree $\gamma$, we conclude that the hypersurface given by

$$
\tilde{f}\left(x_{1}, \ldots, x_{n}\right)=\left(x_{1}, \ldots, x_{n}, \lambda^{\gamma-1} F\left(x_{1}, \ldots, x_{n}\right)\right)
$$

is also minimal. In this case, the minimality condition (46) becomes

$$
\left(\sum_{i=1}^{n} F_{i i}\right)\left(\lambda^{2-2 \gamma}+\sum_{i=1}^{n} F_{i}^{2}\right)=\sum_{i, j} F_{i} F_{j} F_{i j} .
$$

Comparing (49) with (46), we obtain that either $\gamma=1$ or $\gamma \neq 1$ and

$$
\begin{gathered}
\sum_{i=1}^{n} F_{i i}=0, \\
\sum_{i, j} F_{i} F_{j} F_{i j}=0 .
\end{gathered}
$$


Moreover, it follows from (45) that

$$
\begin{gathered}
F_{i}=A \gamma a_{i}^{\rho} x_{i}^{\rho-1}\left(\sum_{i=1}^{n} a_{i}^{\rho} x_{i}^{\rho}\right)^{\gamma / \rho-1} \\
F_{i i}=A \gamma a_{i}^{\rho} x_{i}^{\rho-2}\left((\rho-1) \sum_{k \neq i} a_{k}^{\rho} x_{k}^{\rho}+(\gamma-1) a_{i}^{\rho} x_{i}^{\rho}\right) \\
\times\left(\sum_{i=1}^{n} a_{i}^{\rho} x_{i}^{\rho}\right)^{\gamma / \rho-2}, \\
F_{i j}=A \gamma(\gamma-\rho) a_{i}^{\rho} a_{j}^{\rho} x_{i}^{\rho-1} x_{j}^{\rho-1}\left(\sum_{i=1}^{n} a_{i}^{\rho} x_{i}^{\rho}\right)^{\gamma / \rho-2}, i \neq j .
\end{gathered}
$$

We divide it into two cases.

Case $A(\gamma \neq 1)$. Substituting (52) and (54) into (51), we get

$$
\begin{aligned}
A^{3} \gamma^{3} & (\gamma-\rho)\left(\sum_{i=1}^{n} a_{i}^{\rho} x_{i}^{\rho}\right)^{3 \gamma / \rho-4} \\
\times \sum_{i, j}\left(a_{i}^{2 \rho} a_{j}^{2 \rho} x_{i}^{2 \rho-2} x_{j}^{2 \rho-2}\right) & =0 .
\end{aligned}
$$

If $\rho \neq \gamma$, then the above equation reduces to

$$
\sum_{i, j}\left(a_{i}^{2 \rho} a_{j}^{2 \rho} x_{i}^{2 \rho-2} x_{j}^{2 \rho-2}\right)=0,
$$

which is equivalent to

$$
\left(\sum_{i=1}^{n} a_{i}^{2 \rho} x_{i}^{2 \rho-2}\right)^{2}=0 .
$$

Hence

$$
\sum_{i=1}^{n} a_{i}^{2 \rho} x_{i}^{2 \rho-2}=0
$$

Since $\rho \neq 1$, this case is impossible.

Therefore we conclude that $\rho=\gamma$. Substituting (53) into (50), we have

$$
\begin{aligned}
A \gamma(\gamma & -1)\left(\sum_{i=1}^{n} a_{i}^{\rho} x_{i}^{\rho-2}\right) \\
& \times\left(\sum_{i=1}^{n} a_{i}^{\rho} x_{i}^{\rho}\right)\left(\sum_{i=1}^{n} a_{i}^{\rho} x_{i}^{\rho}\right)^{\gamma / \rho-2}=0 .
\end{aligned}
$$

Noting $\gamma \neq 1$, it is easy to see that (59) is impossible.

Case $B(\gamma=1)$. We note that the minimality condition reduces to

$$
\sum_{i=1}^{n} F_{i i}+\sum_{i \neq j}\left(F_{i}^{2} F_{j j}-F_{i} F_{j} F_{i j}\right)=0 .
$$

Substituting (52)-(54) into (60), we have

$$
\begin{aligned}
& A(\rho-1)\left(\sum_{k \neq i} a_{k}^{\rho} x_{k}^{\rho}\right)\left(\sum_{i=1}^{n} a_{i}^{\rho} x_{i}^{\rho}\right)^{1 / \rho-2}\left(\sum_{i=1}^{n} a_{i}^{\rho} x_{i}^{\rho-2}\right) \\
& +A^{3}(\rho-1)\left(\sum_{k \neq i} a_{k}^{\rho} x_{k}^{\rho}\right)\left(\sum_{i=1}^{n} a_{i}^{\rho} x_{i}^{\rho}\right)^{3 / \rho-4} \\
& \quad \times \sum_{i \neq j}\left(a_{i}^{2 \rho} a_{j}^{\rho} x_{i}^{2 \rho-2} x_{j}^{\rho-2}\right) \\
& -A^{3}(1-\rho)\left(\sum_{i=1}^{n} a_{i}^{\rho} x_{i}^{\rho}\right)^{3 / \rho-4} \\
& \quad \times \sum_{i \neq j}\left(a_{i}^{2 \rho} a_{j}^{2 \rho} x_{i}^{2 \rho-2} x_{j}^{2 \rho-2}\right)=0 .
\end{aligned}
$$

Note that $\rho=1$ fulfills (61). Hence, in this case the generalized CES production function $F$ is a perfect substitute.

In the following, we will show that the case $\rho \neq 1$ is impossible. In fact, if $\rho \neq 1$, (61) reduces to

$$
\begin{gathered}
\left(\sum_{k \neq i} a_{k}^{\rho} x_{k}^{\rho}\right)\left(\sum_{i=1}^{n} a_{i}^{\rho} x_{i}^{\rho}\right)^{2-2 / \rho}\left(\sum_{i=1}^{n} a_{i}^{\rho} x_{i}^{\rho-2}\right) \\
+A^{2}\left(\sum_{k \neq i} a_{k}^{\rho} x_{k}^{\rho}\right) \sum_{i \neq j}\left(a_{i}^{2 \rho} a_{j}^{\rho} x_{i}^{2 \rho-2} x_{j}^{\rho-2}\right) \\
+A^{2} \sum_{i \neq j}\left(a_{i}^{2 \rho} a_{j}^{2 \rho} x_{i}^{2 \rho-2} x_{j}^{2 \rho-2}\right)=0 .
\end{gathered}
$$

Since $x_{i}>0$ and $a_{i}>0$ for $i=1, \ldots, n$, it follows that the left of equality (62) is positive. Therefore, it is a contradiction.

Conversely, it is easy to verify that if the generalized CES production function is a perfect substitute then the $n$-factor generalized CES production hypersurface in $\mathbb{E}^{n+1}$ is minimal. This completes the proof of Theorem 3.

Remark 4. Remark that Chen proved in [7] a more general result for 2-factor: $h$-homogeneous production function is a perfect substitute if and only if the production surface is a minimal surface.

\section{Acknowledgments}

The authors would like to thank the referees for their very valuable comments and suggestions to improve this paper. This work is supported by the Mathematical Tianyuan Youth Fund of China (no. 11326068), the general Financial Grant from the China Postdoctoral Science Foundation (no. 2012M510818), and the General Project for Scientific Research of Liaoning Educational Committee (no. W2012186).

\section{References}

[1] C. W. Cobb and P. H. Douglas, "A theory of production," American Economic Review, vol. 18, pp. 139-165, 1928. 
[2] K. J. Arrow, H. B. Chenery, B. S. Minhas, and R. M. Solow, "Capital-labor substitution and economic efficiency," Review of Economics and Statistics, vol. 43, no. 3, pp. 225-250, 1961.

[3] L. Losonczi, "Production functions having the CES property," Academiae Paedagogicae Nyíregyháziensis, vol. 26, no. 1, pp. 113$125,2010$.

[4] D. McFadden, "Constant elasticity of substitution production functions," Review of Economic Studies, vol. 30, no. 2, pp. 73-83, 1963.

[5] G. E. Vîlcu, "A geometric perspective on the generalized CobbDouglas production functions," Applied Mathematics Letters, vol. 24, no. 5, pp. 777-783, 2011.

[6] A. D. Vîlcu and G. E. Vîlcu, "On some geometric properties of the generalized CES production functions," Applied Mathematics and Computation, vol. 218, no. 1, pp. 124-129, 2011.

[7] B.-Y. Chen, "On some geometric properties of $h$-homogeneous production functions in microeconomics," Kragujevac Journal of Mathematics, vol. 35, no. 3, pp. 343-357, 2011.

[8] B.-Y. Chen, "On some geometric properties of quasi-sum production models," Journal of Mathematical Analysis and Applications, vol. 392, no. 2, pp. 192-199, 2012.

[9] B.-Y. Chen, "A note on homogeneous production models," Kragujevac Journal of Mathematics, vol. 36, no. 1, pp. 41-43, 2012.

[10] B.-Y. Chen, "Geometry of quasi-sum production functions with constant elasticity of substitution property," Journal of Advanced Mathematical Studies, vol. 5, no. 2, pp. 90-97, 2012.

[11] B.-Y. Chen, "Classification of homothetic functions with constant elasticity of substitution and its geometric applications," International Electronic Journal of Geometry, vol. 5, no. 2, pp. 67-78, 2012.

[12] B.-Y. Chen, "Solutions to homogeneous Monge-Ampère equations of homothetic functions and their applications to production models in economics," Journal of Mathematical Analysis and Applications, vol. 411, no. 1, pp. 223-229, 2014.

[13] B.-Y. Chen and G. E. Vîlcu, "Geometric classifications of homogeneous production functions," Applied Mathematics and Computation, vol. 225, pp. 345-351, 2013.

[14] A. D. Vilcu and G. E. Vilcu, "On homogeneous production functions with proportional marginal rate of substitution," Mathematical Problems in Engineering, vol. 2013, Article ID 732643, 5 pages, 2013.

[15] B.-Y. Chen, Pseudo-Riemannian Geometry, $\delta$-Invariants and Applications, World Scientific, Hackensack, NJ, USA, 2011.

[16] B.-y. Chen, Geometry of Submanifolds, Marcel Dekker, New York, NY, USA, 1973. 


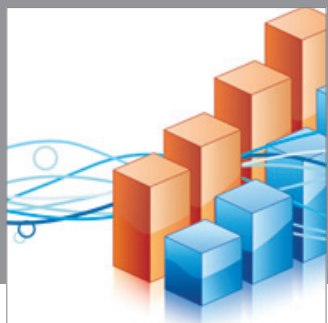

Advances in

Operations Research

mansans

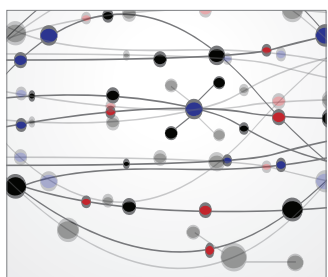

The Scientific World Journal
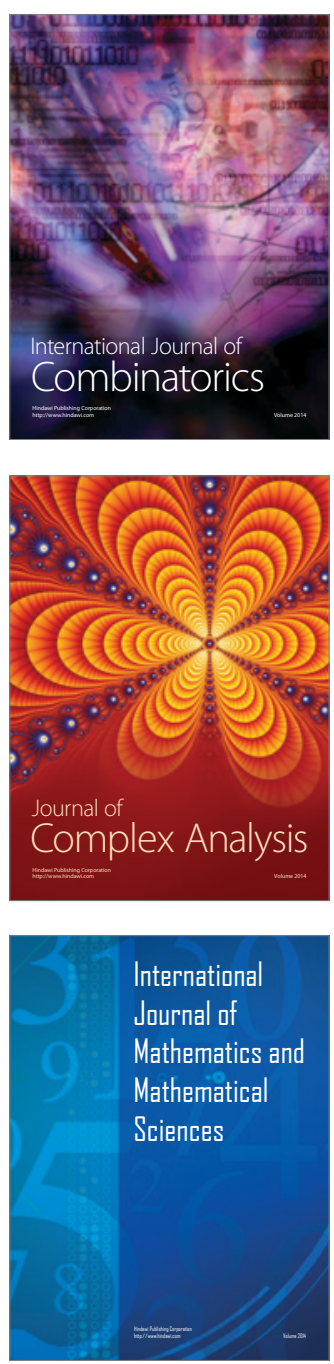
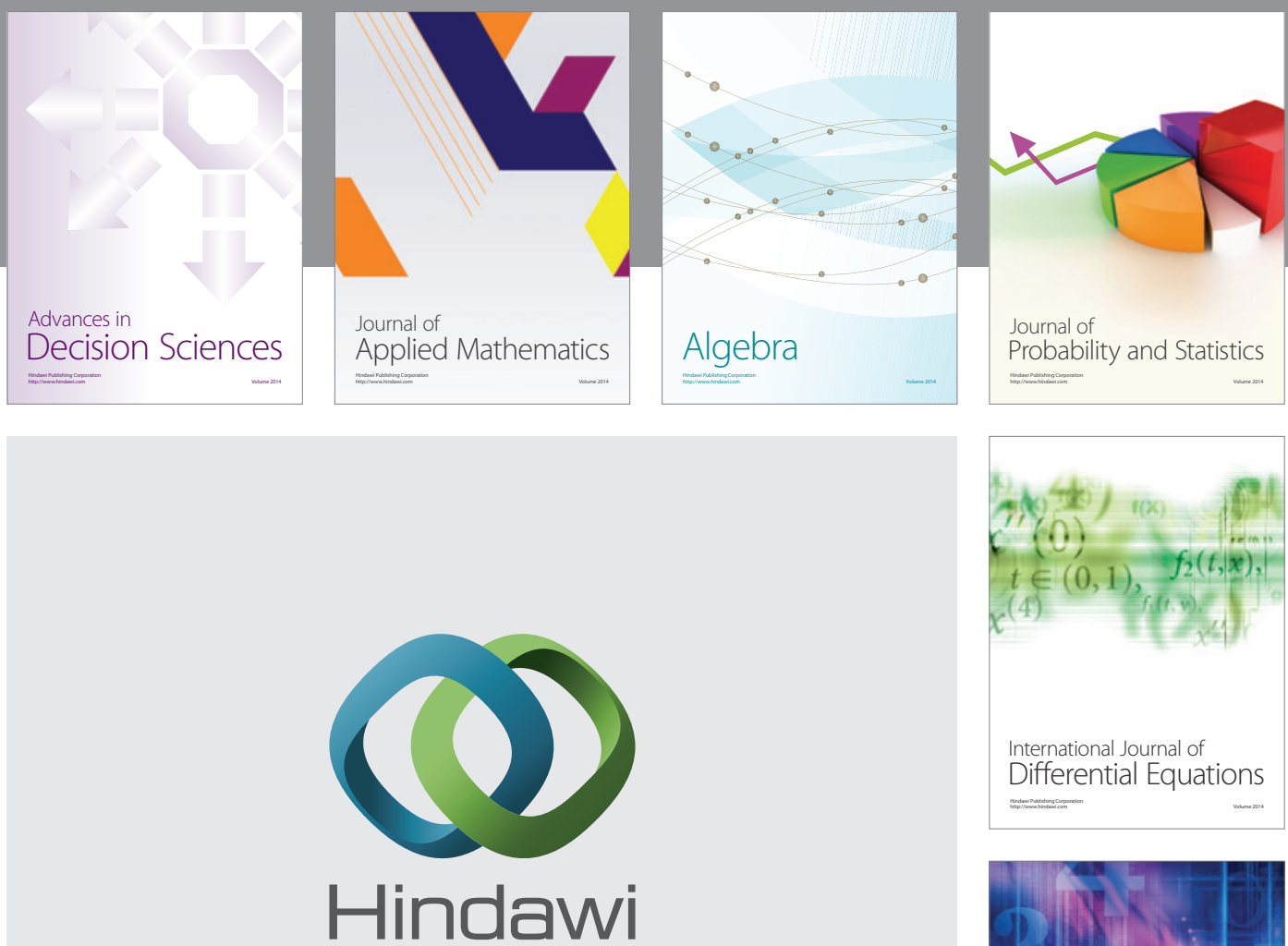

Submit your manuscripts at http://www.hindawi.com
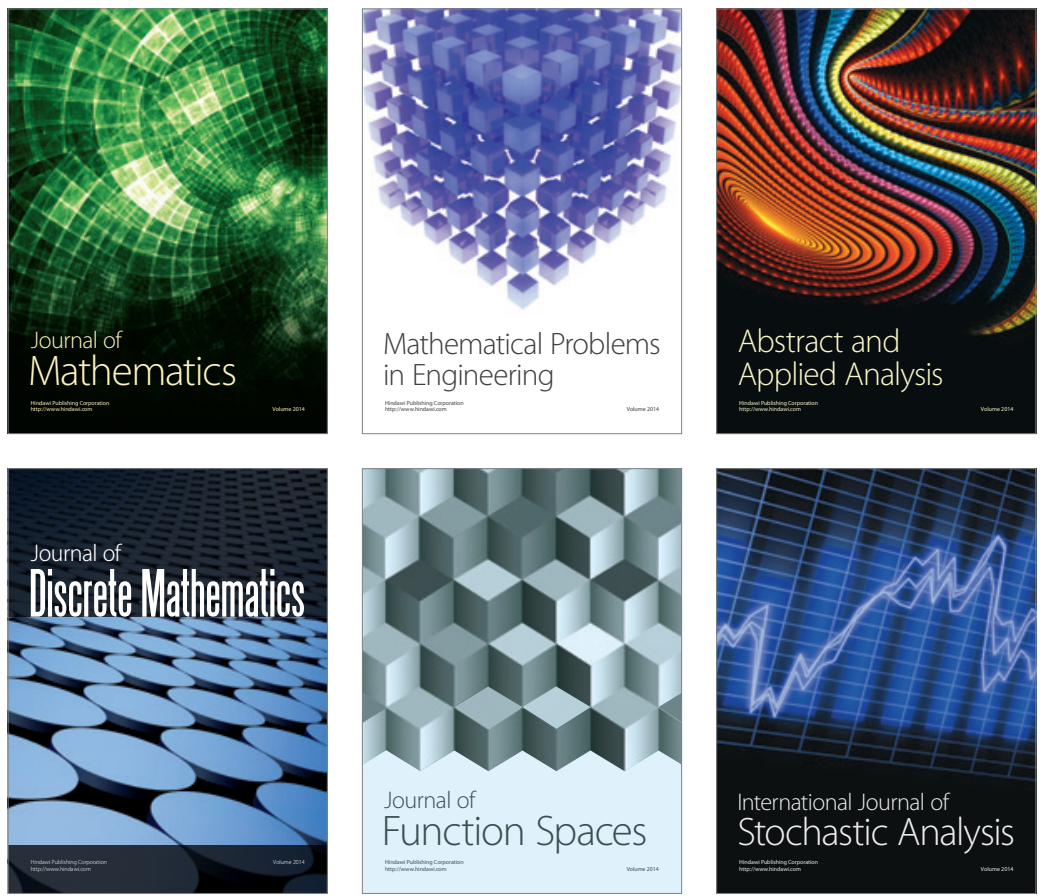

Journal of

Function Spaces

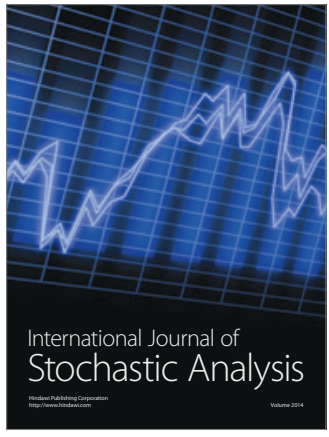

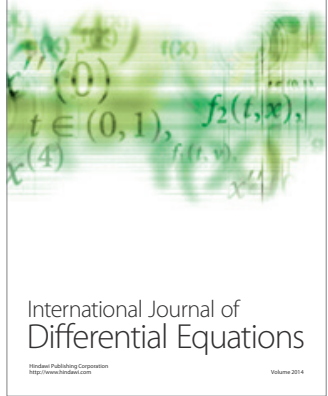
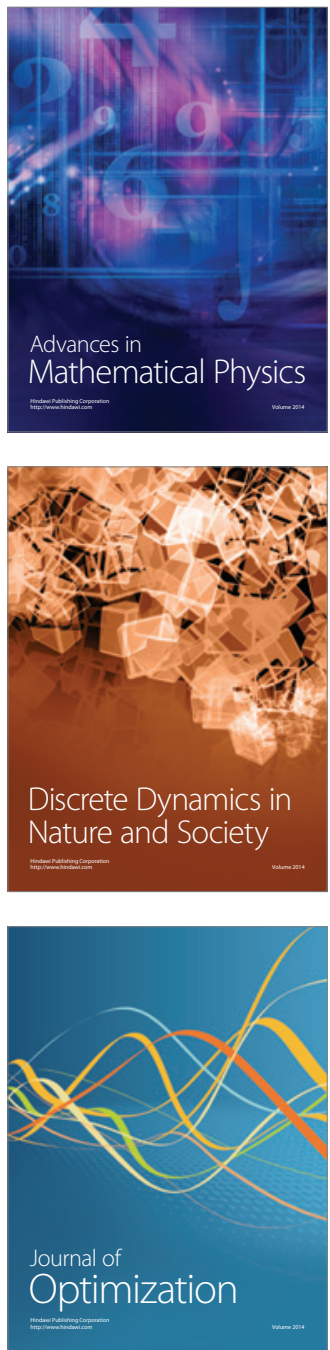